\title{
Variantes anatómicas de la segmentación bronquial, preva- lencia y diagnóstico mediante TC multidetector 64 canales
}

\author{
Dres. Mariana Robledo M, Carlos Bertona T, Juan Bertona T, Cecilia Giordanengo M, Noelia Gribaudo C, Irina Tolabin S.
}

Clínica Privada Vélez Sarsfield, Córdoba, Argentina.

Bronchial segmentation: Anatomical variants, prevalence, and diagnosis by 64-channel multidetector CT

\begin{abstract}
Purpose. A) To determine types and prevalence of anatomical variants of bronchial segmentation in patients who underwent 64-section Multidetector CT (MDCT) of the chest. B) To evaluate the ability of this method as a diagnostic tool. Material and methods. We conducted a retrospective review of chest computed tomography studies of 156 adult patients, performed with Toshiba, Aquilion 64 CFX, CT Scanner Equipment, with chest volumetric acquisitions, and cuts of $0.5 \mathrm{~mm}$. Images obtained allowed MPR and 3D reconstructions, along with virtual endoscopic visualization of the tracheobronchial tree, in the study period from may 2007 to may 2011, irrespective of reasons to request those examinations. Results. Eighty-nine (57\%) of the 156 cases corresponded to male patients and 67 (43\%) to female.patients. Fifty-two variants were found in 47 patients, since in five of them two variants were observed. The most commonly encountered variants were: variant of number (28 variants, 53\%), of which 15 (28\%) showed sub-superior lobar bronchus, 6 (11\%) accessory bronchus in the left lower lobe; $5(10 \%)$ isomerism of lower lobes, and $2(4 \%)$ accessory bronchus in the right upper lobe. Among variations of origin (24 variants, 47\%) we found 17 variants (33\%) with right upper lobe bifurcation; 6 (12\%) exhibited trifurcation of the left upper lobe bronchus, and 1 (2\%) showed accessory tracheal bronchus. Conclusion. Anatomical variants in the tracheobronchial tree exhibited a prevalence of $33 \%$ in our study population. We found 52 variants in 47 patients, the most common being bifurcation of the right upper lobe bronchus, and subsuperior lobar bronchus. The study of anatomical variants, along with total anatomy of the tracheobronchial tree by $64 \mathrm{MDCT}$, irrespective of study protocol applied, can provide useful information prior to performing more invasive procedures, such as conventional bronchoscopy.
\end{abstract}

Key words: Anatomical variants, Bronchial segmentation, Multidetector tomografia, Virtual bronchoscopy.

Resumen: Propósito. Conocer tipos y prevalencia de las variantes anatómicas de la segmentación bronquial, en pacientes a quienes se les realizó TC de Tórax Multidetector 64 (TCMD 64). Evaluar la capacidad de este método para el diagnóstico de las mismas. Material y métodos. Se estudiaron en forma retrospectiva los estudios tomográficos que incluyeron el tórax, en 156 pacientes adultos, con tomógrafo Toshiba Aquilion 64, con adquisiciones volumétricas del tórax, cortes de $0.5 \mathrm{~mm}$, obteniendo imágenes que permitieron reconstrucciones MPR, 3D y visualización endoscópica virtual traqueobronquial, en el período entre mayo de 2007 y mayo de 2011, independientemente del motivo por el cual fueron solicitados. Resultados. De los 156 estudios examinados, 89 (57\%) fueron de sexo masculino y 67 (43\%) femenino. En su análisis se encontraron 52 variantes, en 47 pacientes, ya que en cinco de ellos se observaron dos variantes. Entre las más frecuentes fueron: de número (28 variantes-53\%), de las cuales: 15 (28\%) con bronquio sub-superior; 6 (11\%) bronquio accesorio en lóbulo inferior izquierdo; 5 (10\%) isomerismo de lóbulos inferiores; y 2 (4\%) bronquio accesorio en lóbulo superior derecho. Entre las variantes de origen (24 variantes-47\%) encontramos 17 variantes (33\%) con bifurcación del lóbulo superior derecho; 6 (12\%) trifurcación del bronquio del lóbulo superior izquierdo; y 1 (2\%) bronquio traqueal accesorio. Conclusión. Las variantes anatómicas en el árbol traqueobronquial presentaron una prevalencia en nuestra población de estudio del 33\%, hallándose 52 en 47 pacientes, siendo las más frecuentes la bifurcación del lóbulo superior derecho y el bronquio subsuperior. Su estudio y el de la anatomía total del árbol traqueo-bronquial mediante TCMD 64, independientemente del protocolo de estudio empleado, puede aportar información de utilidad previo a la realización de procedimientos más invasivos como la broncoscopía convencional.

Palabras clave: Broncoscopía virtual, Segmentación bronquial, Tomografía multidetector, Variantes anatómicas.

Bertona $C$, et al. Variantes anatómicas de la segmentación bronquial, prevalencia y diagnóstico mediante TC multidetector 64 canales. Rev Chil Radiol 2012; 18(4): 170-174.

Correspondencia: Dra. Mariana Robledo M. / mara_r_@hotmail.com

Trabajo recibido el 22 de noviembre de 2012, aceptado para publicación el 27 de noviembre de 2012. 


\section{Introducción}

El estudio del árbol traqueobronquial y sus numerosos patrones de variantes de ramificaciones, ha sido de gran interés anatómico y quirúrgico a lo largo de la historia.

El abordaje en el estudio de la segmentación bronquial comenzó en el año 1880 con los estudios de Aeby y la realización de disecciones en mamíferos, tomando tres enunciados principales: bronquios principales, régimen monopódico y de los sistemas bronquiales, sin profundizar su estudio en las variantes normales. Luego, en 1943, Jackson y Huber continuaron su investigación con la clasificación de los bronquios segmentarios según su orientación y encontraron variaciones de fusión de troncos o por agenesia. En el transcurso del tiempo el estudio de las variantes del árbol traqueobronquial fue cobrando mayor interés. Ghaye en el año 2001, realizó un estudio mediante un tomógrafo helicoidal, con reconstrucción multiplanar (RMP) y tridimensionales (3D) de los bronquios; determinando las siguientes variaciones: bronquios supernumerarios, agenesiashipoplasias y desplazamientos bronquiales. Saladrigas Sarduy en el año 2008 analiza las variaciones de número (supernumerarios, agenesias-hipoplasias) $y$ de origen (desplazamientos) $)^{(1,2)}$.

El estudio por imágenes de las vías aéreas provee información de utilidad para el diagnóstico y toma de decisiones terapéuticas. En los últimos años, la tomografía computada helicoidal con múltiples detectores (TCMD) ha progresado enormemente. En particular, el reciente avance en el campo de 64 cortes en un tiempo de 330 milisegundos por rotación, constituye una técnica de imagen de alta resolución espacial y temporal. La información adquirida con TCMD, puede ser utilizada para la realización RMP de alta calidad orientadas a lo largo de cualquier eje deseado, reconstrucción 3D e imágenes de la luz de las vías aéreas por broncoscopía virtual $(\mathrm{BV})^{(3)}$.

La BV es un procedimiento no invasivo de gran utilidad para el diagnóstico y seguimiento de las enfermedades del aparato respiratorio que requiere de un profundo conocimiento de la anatomía del árbol bronquial normal, sus variantes más comunes y sus relaciones anatómicas ${ }^{(4)}$.

La habilidad del médico imagenólogo en la evaluación de las BV, para identificar anormalidades en el árbol traqueobronquial, obvias y no, puede influir en el protocolo de estudio y/o manejo de los pacientes.

El presente trabajo tiene como objetivo conocer el tipo y la prevalencia de las variantes anatómicas de la segmentación bronquial, en pacientes a quienes se les realizó TCMD 64 en nuestro servicio y de esa forma evaluar la capacidad de este método para el diagnóstico de las mismas.

\section{Materiales y métodos}

Se efectuó un estudio descriptivo en el que se analizaron retrospectivamente estudios tomográficos de tórax, realizados entre mayo de 2007 y mayo de 2011, en nuestra institución.

Muestra: En el estudio se incluyeron todos los estudios tomográficos de tórax, independientemente del motivo por el cual fueron solicitados.

Técnica: Para las exploraciones se utilizó un tomógrafo Toshiba Aquilion 64, con adquisiciones volumétricas de tórax, con cortes de $0,5 \mathrm{~mm}$. El análisis de la información se realiza en una estación de trabajo Vitrea mediante MPR, MinIP, 3D y BV traqueobronquial.

Variables examinadas: Los datos se recolectaron en dos planillas, diseñadas a tal fin en donde se consideraron las siguientes:

\section{- Sexo}

- Segmentación pulmonar normal

- Variantes de número: bronquio sub-superior, bronquio accesorio en lóbulo inferior izquierdo, isomerismo en lóbulos inferiores y bronquio accesorio en lóbulo superior derecho,

- Variantes de origen: bifurcación del lóbulo superior derecho, trifurcación del lóbulo superior izquierdo y bronquio traqueal accesorio.

\section{Resultados}

De los 156 estudios examinados, 89 (57\%) fueron de sexo masculino y 67 (43\%) femenino (Gráfico 1).

En su análisis se evaluaron 52 variantes normales (33\%) (Gráfico 3) en 47 pacientes por presentar doble hallazgo en cinco de ellos. De las variantes normales se observaron:

- $28(53 \%)$ variantes de número: de las cuales $15(28 \%)$ con bronquio sub-superior, $6(11 \%)$ bronquio accesorio en lóbulo inferior izquierdo, 5 (10\%) isomerismo de lóbulos inferiores y $2(4 \%)$ bronquio accesorio del lóbulo superior derecho. (Figuras 2, 3, 4 y 5) (Gráfico 4).

- $24(47 \%)$ variantes de origen: $17(33 \%)$ variantes con bifurcación del lóbulo superior derecho, 6 (12\%) trifurcación del lóbulo superior izquierdo y $1(2 \%)$ bronquio traqueal accesorio. (Figuras 1 , 3, 4, 6 y 7) (Gráfico 5).

\section{Discusión}

Los resultados obtenidos en nuestro trabajo nos permitieron arribar a las siguientes conclusiones principalmente con respecto a las variaciones:

1) En el estudio de nuestra muestra, al igual que en la bibliografía consultada, las variaciones bronquiales son frecuentes, observándose en el $33 \%$.

2) Se pudo observar la presencia de variantes bronquiales, tanto de número como de origen en igual frecuencia. 
3) Entre las variantes de origen se observó con más frecuencia la bifurcación del lóbulo superior derecho y en el $2 \%$ bronquio traqueal, accesorio que coincide con la bibliografía consultada.

4) Diferente a lo referido en las publicaciones revisadas, entre las variantes de número no se registraron agenesias segmentarias.

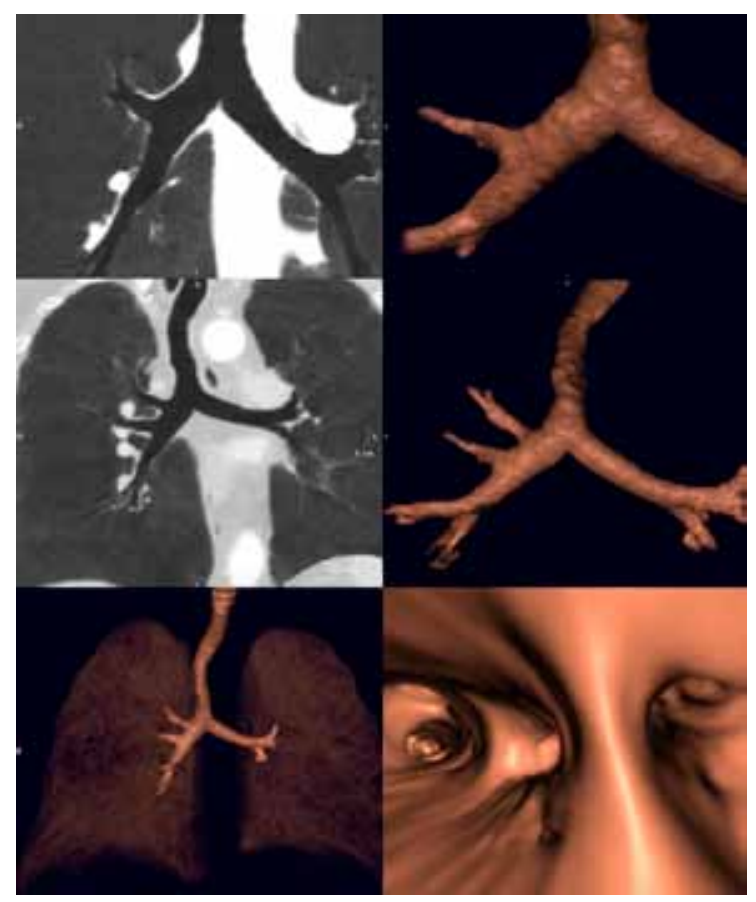

Figura 1. Bronquio accesorio del lóbulo superior derecho.

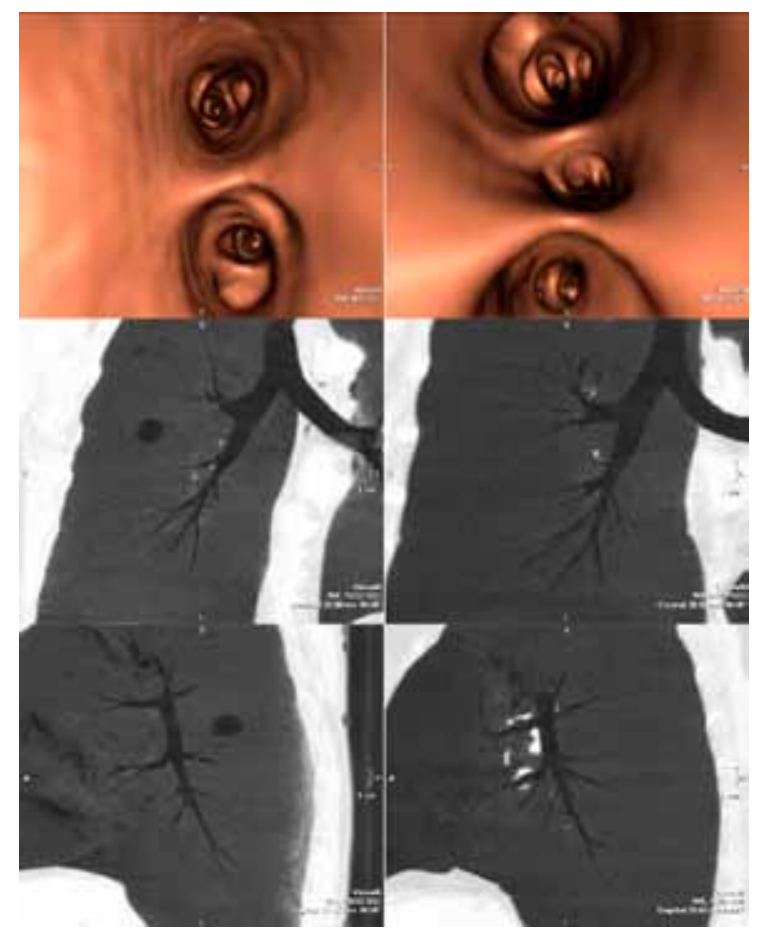

Figura 2. Bronquio sub-superior.

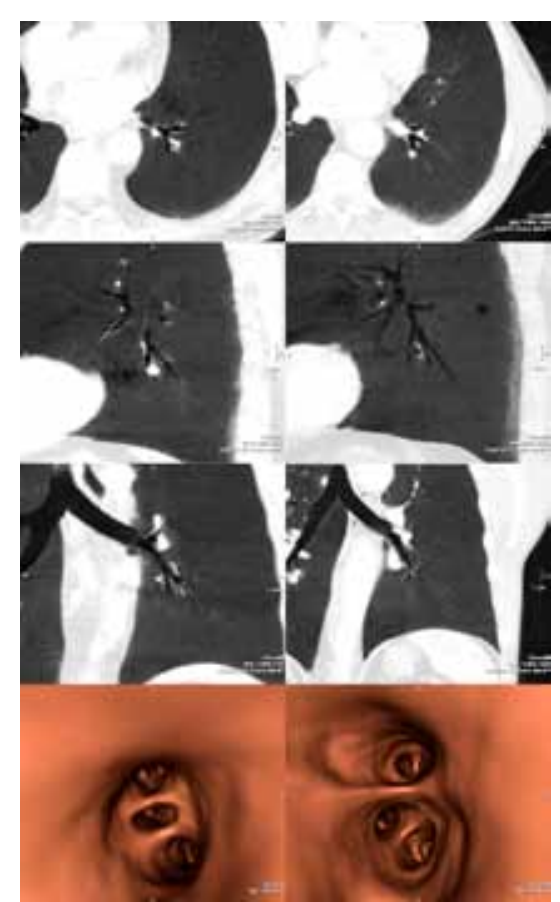

Figura 3. Bronquio accesorio del lóbulo inferior izquierdo.

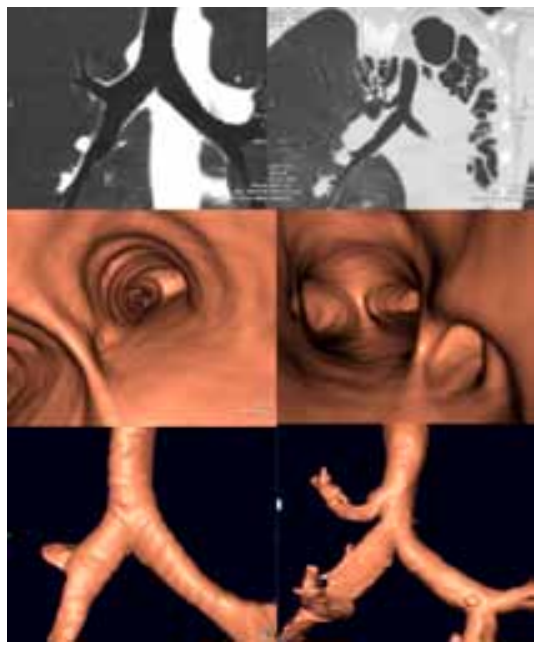

Figura 4. Bronquio traqueal accesorio.
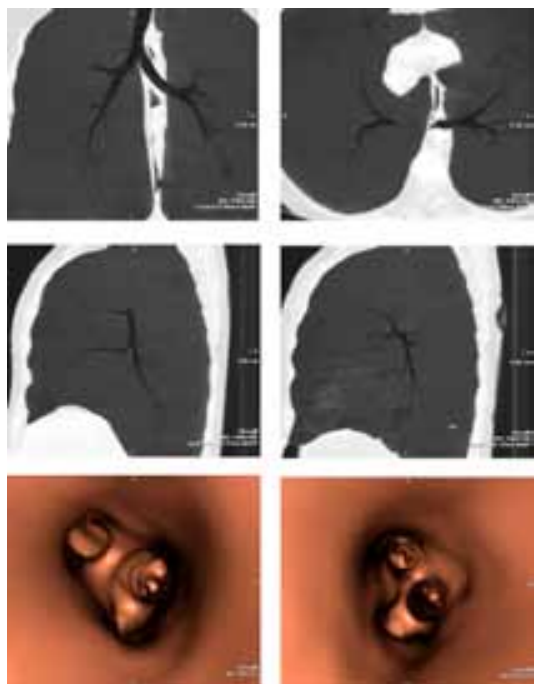

Figura 5. Isomerismo de lóbulos inferiores. 


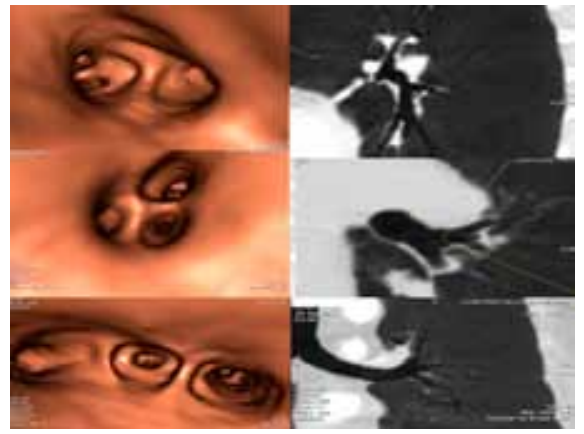

Figura 6. Trifurcación del lóbulo superior izquierdo.

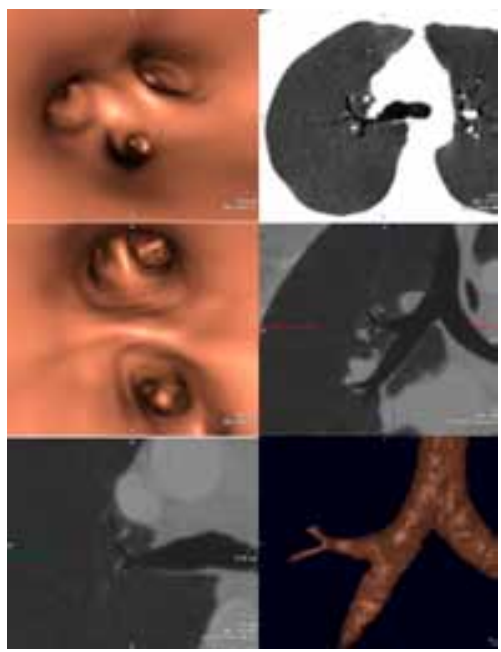

Figura 7. Bifurcación del lóbulo superior derecho.
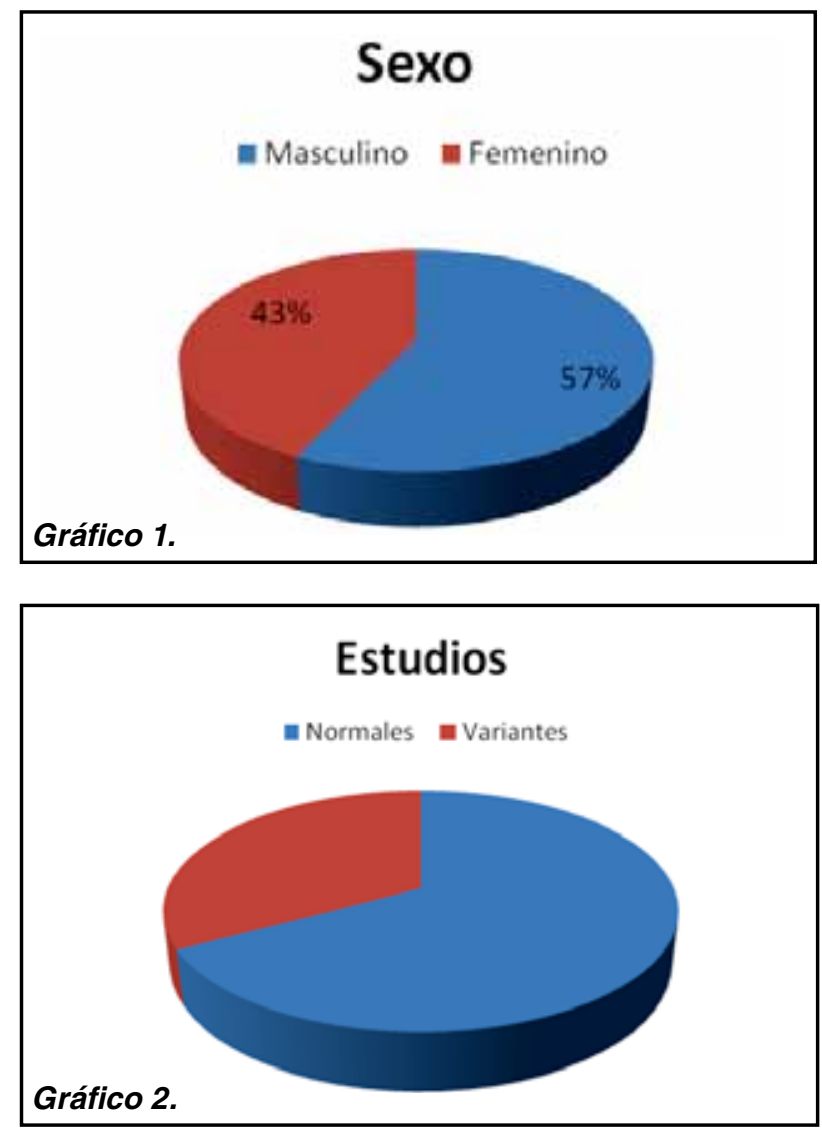

Tipos de Variantes Normales

número = Origen

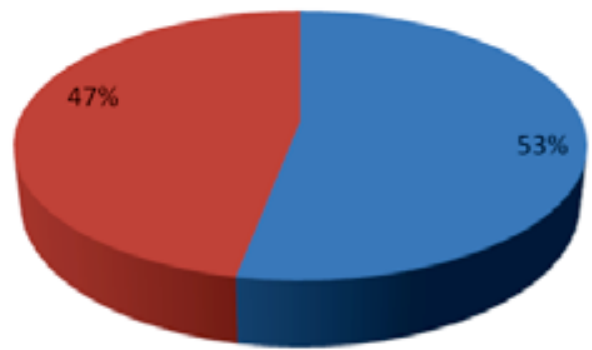

Gráfico 3.
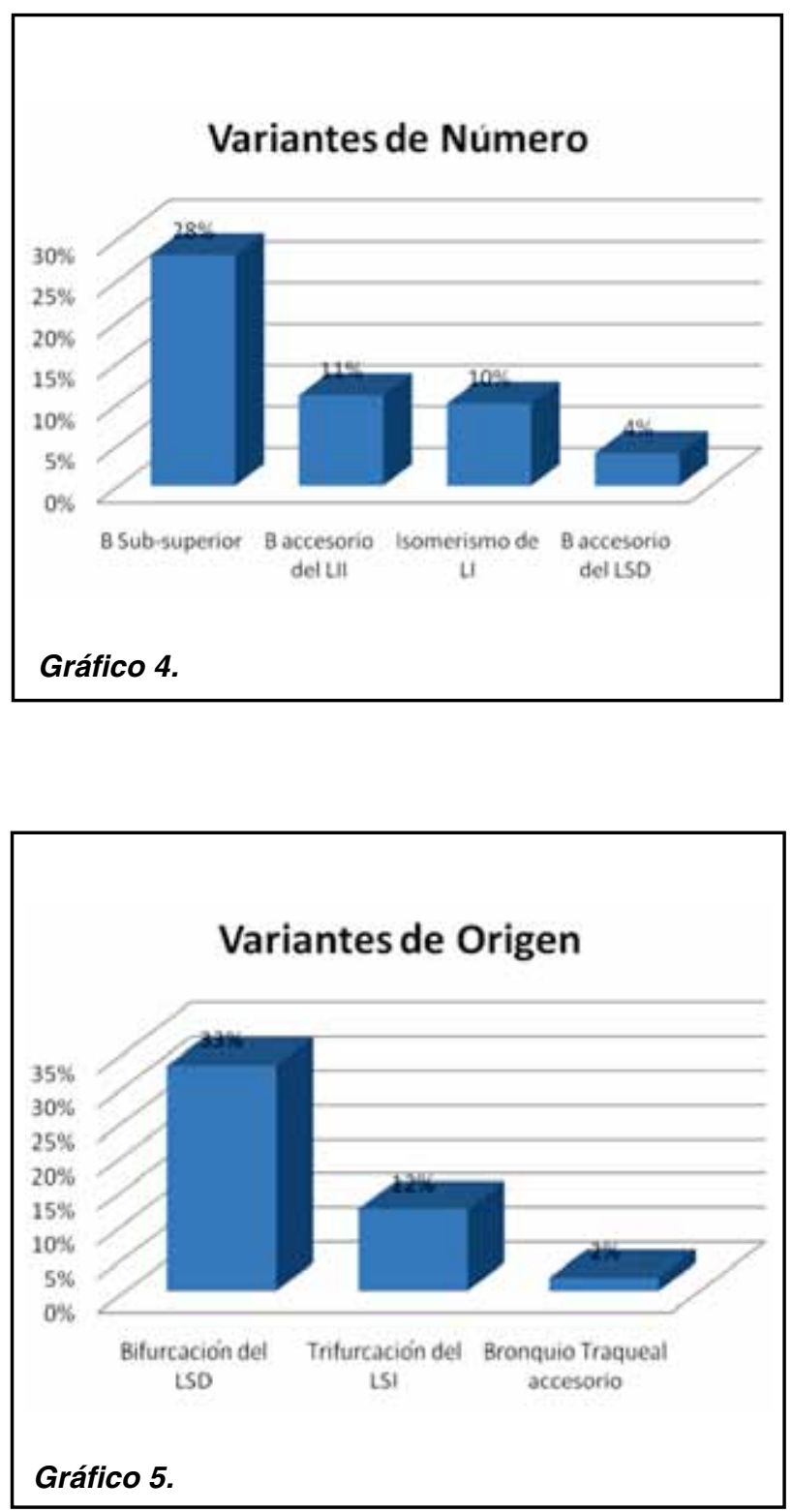
La tomografía computada es hoy en día el método de elección para valorar la vía aérea. La TCMD tiene la ventaja a través de RMP, valorar estructuras vasculares y bronquiales en distintas dimensiones, la calidad de la RMP (coronal, sagital y curva), con 3D, permite la rotación optima de la imagen, facilita el análisis de puntos específicos y precisa información anatómica y de sus variantes. Mediante la BV es posible recorrer la luz de tráquea, bronquios principales, lobares y segmentarios, para valorar patologías intrínsecas como también variantes anatómicas de la segmentación.

Concluimos en nuestro trabajo que el estudio de la anatomía total del árbol taqueobronquial y de sus variantes normales mediante TCMD 64, puede aportar información de utilidad previo a la realización de procedimientos más invasivos como la broncoscopía convencional.

\section{Bibliografía}

1. Ghaye B, Szapiro D, Fanchamps JM, Dondelinger RF. Congenital Bronchial Anormalities Revistied. Radiographics J. 2001; 21: 105-119.

2. Testut L, Latarjet A. Tratado de Anatomía Humana: Tomo III. Novena Edición, Salvat 1975; p 959-972.

3. Netter, F. Atlas of Human Anatomy. 2th Ed. Canada: Learning System; 2001; p. 191.

4. Kazerooni E, Gross B. Radiología Cardiopulmonar. Edición Journal. 2006; 16: 433-437.

5. Fraser RS, Colman N, Müller NL, Paré PD. Diagnóstico de las Enfermedades del Tórax. 4ta. edición. Vol. I 2006;p. 620-623. 